\title{
Das jahresperiodische Verhalten der Fettsäuren in Rinden und Blättern von Pinus halepensis Mill. und Schinus molle L.
}

\author{
Fatty Acid Composition of Barks and Leaves of Pinus halepensis Mill. \\ and Schinus molle L. During the Course of a Year
}

\section{S. Diamantoglou und U. Kull}

Institut für Allgemeine Botanik der Universität Athen 621, Griechenland und Biologisches Institut der Universität Stuttgart, Ulmer Str. 227, D-7000 Stuttgart, F.R.G.

Eingegangen am 8. Dezember 1980 - Angenommen am 6. März 1981

\section{Summary}

The fatty acid composition of the saponifiable lipids from barks and leaves of Pinus balepensis and of Schinus molle was investigated throughout the period of a year by gas liquid chromatography. Both species showed a great variety of different fatty acids of saponifiable lipids. In barks of Pinus, the contents of stearic, oleic and linoleic acids are higher during late winter and the growth period, whereas the percentage of behenic, lignocerinic, octadecatetraenoic, eicosatrienoic, and eicosatetraenoic acids was larger during the summer drought period and the autumn months. In the needles of Pinus, the contents of linolenic acid and of the short-chain acids (caprinic and laurinic) showed maxima during the winter and the growth period. In barks of Schinus we found a larger fraction of linolenic, erucic, and lignocerinic acids during the same period; in the leaves of this species linoleic, linolenic, behenic, and lignocerinic acids accumulated during the winter.

There is no unequivocal influence of summer-drought on fatty acid pattern. In all species of linoleic and linolenic acids the highest percentage is shown during the humid winter and growth periods, which seems to reflect the metabolic activity of the investigated tissues.

Key words: Pinus halepensis, Schinus molle, fatty acid compositıon, temperature, drought.

\section{Einleitung}

Die Jahresperiodik der Fettsäure-Zusammensetzung von Holzpflanzen des Mittelmeergebiets wurde bisher an Arten mit einfachen Fettsäure-Mustern untersucht, so bei Olea europaea (Catalano und Sciancalepore, 1975; Donaire und LopezGorgé, 1978), Ceratonia siliqua (Diamantoglou und Meletiou-Christou, 1977) und an mehreren Arten der Gattung Pistacia (Diamantoglou und Meletiou-Christou, 1979). Wir haben die Untersuchungen nun auf zwei Arten mit erheblich komplexerer 
Fettsäure-Zusammensetzung der verseifbaren Lipide ausgedehnt. Pinus halepensis, der für die mediterrane Vegetation wichtigste Nadelbaum, besitzt ein FettsäureMuster, das demjenigen anderer Pinus-Arten (Porter, 1969; Jamieson und Reid, 1972) weitgehend entspricht. Schinus molle stammt aus Südamerika, wird aber im Mittelmeergebiet häufig gepflanzt. Diese Art wurde herangezogen, weil sie wie die kürzlich untersuchten Pistacia-Arten zu den Anacardiaceae gehört. Von Schinus ist ein Vorkommen mehrerer sehr langkettiger Fettsäuren in Früchten bekannt (PozzoBalbi et al., 1978). Unsere Untersuchungen zeigen, daß in vegetativen Teilen eine große Zahl verschiedener Fettsäuren in der Lipidfraktion vorkommt.

\section{Material und Methoden}

\section{Material}

Untersucht wurden Rinden und Blätter von Pinus halepensis Mill. und Schinus molle L. Das Material wurde jeweils einem etwa 50jährigen Baum entnommen. Beide Bäume wachsen in einem offenen Gelände nahe dem Botanischen Institut der Universität Athen und werden nicht künstlich bewässert. Das Material wurde in der Mitte jeden Monats zur selben Tageszeit (ca. $10 \mathrm{Uhr}$ ) geerntet, um tagesperiodische Schwankungen möglichst gering zu halten. Die abgeschälte Rinde und die adulten Blätter (bei Pinus einjährige Nadeln) wurden in Plastikbeuteln $10 \mathrm{~min}$ lang in kochendem Wasser gehalten und anschließend bei $60^{\circ} \mathrm{C}$ in einem Ventilatortrockenschrank getrocknet.

Temperatur- und Niederschlagsverhältnisse des Standortes im Untersuchungszeitraum sind bei Diamantoglou (1980) dargestellt.

\section{Methoden}

Die Extraktion der Lipide, die Verseifung und Veresterung erfolgten wie bei Kull und Jeremias (1972) beschrieben. Gaschromatographie: Varian Aerograph 2700, Säule $20^{\prime} \times$ $1 / 8$ " Stahl, $10 \%$ EGSS-X auf Chrom. 60-80 W/AW-DMCS; Temperaturprogramm 172$220^{\circ}$; $2^{\circ}$ je min, Detektor: FID, $280^{\circ} \mathrm{C}$; Injektor: $230^{\circ} \mathrm{C}$; Trägergas: $\mathrm{N}_{2}, 35 \mathrm{ml} / \mathrm{min}$. Die Identifizierung der Peaks und die quantitative Auswertung erfolgten wie bei Höllwarth und Kull (1979) dargestellt mit dem Chromatographie-Datensystem Varian CDS 111. Jede Probe wurde mindestens viermal chromatographiert und die erhaltenen Flächenwerte gemittelt. Als innerer Standard diente der Pentadekansäure-Methylester. Unterschiede von über 1,5\% dürfen als signifikant angesehen werden (vgl. Wiedmaier und Kull, 1978).

$\mathrm{Da}$ von mehreren der langkettigen Fettsäuren $\left(>\mathrm{C}_{20}\right)$ Vergleichssubstanzen nicht zur Verfügung standen, war bei diesen nicht in allen Fällen eine sichere Zuordnung der Peaks möglich. Trotz dem verwendeten Temperaturprogramm ist bei den Fettsäuren mit sehr langen Retentionszeiten ( $a b \mathrm{C}_{22}$ ) die Meßgenauigkeit geringer, Schwankungen von weniger als $3 \%$ sind bei diesen Säuren daher nicht signifikant.

\section{Ergebnisse}

Pinus halepensis

a) Rinde

In den Rinden treten alle geradzahligen gesättigten Fettsäuren von Caprinsäure $(10: 0)$ bis Lignocerinsäure $(24: 0)$ auf (Tabelle 1); davon ist allerdings Arachidin- 
säure $(20: 0)$ nur in Spuren (stets unter $1 \%$ ) nachzuweisen und daher nicht mit in die Tabelle aufgenommen. Ferner kommen verschiedene ungesättigte Fettsäuren mit 14C- bis 20C-Atomen vor (Tabelle 1). Das Fettsäure-Muster entspricht damit den Befunden von Porter (1969) für Pinus radiata. Fettsäuren mit längeren Retentionszeiten als Pentakosansäure traten auf, konnten aber nicht identifiziert werden. Nicht in die Tabelle aufgenommen sind außer der Arachidinsäure noch mehrere andere Fettsäuren mittlerer Kettenlängen, die stets nur unter 1\% am Gesamtfettsäure-Gehalt beteiligt waren.

Table 1: Fatty acid composition of saponifiable lipids from bark of Ptnus halepensis (as \% of total fatty acids) $\mathrm{Sp}=\operatorname{trace}(<0.1 \%)$.

\begin{tabular}{|c|c|c|c|c|c|c|c|c|c|c|c|c|c|}
\hline \multirow[b]{2}{*}{ fatty acid } & \multicolumn{13}{|c|}{ month } \\
\hline & 6 & 7 & 8 & 9 & 10 & 11 & 12 & 1 & 2 & 3 & 4 & 5 & 6 \\
\hline $10: 0$ and $12: 0$ & 0.7 & 1.1 & 0.4 & 1.8 & 0.2 & 0.4 & 0.6 & 0.6 & 2.5 & 1.3 & 0.9 & 1.3 & 1.3 \\
\hline $14: 0$ & 1.0 & 1.7 & 1.0 & 1.5 & 0.9 & 1.0 & 1.3 & 1.4 & 2.8 & 1.7 & 1.2 & 1.2 & 0.3 \\
\hline $16: 0$ & 6.3 & 7.6 & 3.5 & 5.2 & 3.4 & 3.1 & 2.5 & 6.8 & 11.0 & 9.4 & 7.9 & 8.1 & 5.0 \\
\hline $16: u$ & 2.0 & 0.4 & 1.9 & 0.9 & $\mathrm{Sp}$ & $\mathrm{Sp}$ & 0.6 & 0.3 & 1.6 & 0.8 & $\mathrm{Sp}$ & 1.1 & 0.2 \\
\hline $18: 0 \mathrm{al}$ & 22.6 & 17.7 & 9.4 & 6.4 & 7.2 & 7.8 & 11.5 & 23.1 & 25.4 & 23.5 & 19.6 & 23.4 & 19.3 \\
\hline $18: 2$ & 16.1 & 11.8 & 7.4 & 5.4 & 6.1 & 6.9 & 8.1 & 14.7 & 20.6 & 13.9 & 13.7 & 13.2 & 13.8 \\
\hline $18: 3$ & 6.3 & 4.9 & 3.6 & 2.9 & 2.8 & 3.7 & 5.9 & 3.3 & 6.8 & 3.5 & 5.3 & 5.0 & 4.9 \\
\hline $18: 4$ & 5.9 & 6.2 & 6.4 & 4.2 & 4.7 & 4.3 & 8.4 & 1.2 & 3.2 & 1.1 & 4.8 & 2.5 & 6.0 \\
\hline $20: 3$ & 6.1 & 11.0 & 11.9 & 10.9 & 14.6 & 12.4 & 11.5 & 11.5 & 3.1 & 7.0 & 8.9 & 5.1 & 7.2 \\
\hline $20: 4$ & - & - & 4.9 & 6.8 & 4.6 & 2.8 & 7.2 & 0.9 & - & - & 2.5 & - & 2.9 \\
\hline $22: 0$ & 5.8 & 1.8 & 6.7 & 5.3 & 9.0 & 8.5 & 6.1 & - & - & - & - & - & 6.8 \\
\hline $24: 0$ & 10.5 & 14.5 & 14.4 & 16.8 & 17.1 & 16.4 & 9.5 & 10.0 & 8.5 & 14.2 & 12.1 & 9.8 & 13.7 \\
\hline $\begin{array}{l}\text { not identified } \\
(6 \text { peaks })\end{array}$ & 16.7 & 21.5 & 28.5 & 31.8 & 29.4 & 32.7 & 26.8 & 26.2 & 14.5 & 23.7 & 23.1 & 255 & 17.8 \\
\hline
\end{tabular}

Die meisten mengenmäßig bedeutsamen Fettsäuren zeigen deutliche jahresperiodische Schwankungen. Palmitinsäure, Stearin- und Olsäure und Linolsäure haben während der Wintermonate ab Januar und der Wachstumsphase bis zum Sommer höhere Anteile als während der Sommer- und Herbstmonate. Bei der Linolensäure sind die Schwankungen ähnlich, aber etwas unregelmäßiger. Hingegen sind die prozentualen Anteile von Behensäure, Lignocerinsäure sowie der mehrfach ungesättigten Säuren Oktadekatetraensäure, Eikosatrien- und -tetraensäure während der Sommermonate und bis zum Spätherbst erhöht (Tab. 1).

Die Fettsäuren mit mehr als 20C-Atomen können auch Bestandteil der Wachsester und möglicherweise des Harzes sein. Berücksichtigt man aus diesem Grunde diese Fettsäuren bei Berechnung der prozentualen Anteile nicht, so ändert sich das Bild der Jahresperiodik der Fettsäuren etwas. Eine eingreifende Veränderung ist aber nur bei Palmitinsäure vorhanden; sie hat dann während der Sommermonate höhere Anteile und das Minimum liegt im Dezember. 


\section{b) Nadeln}

Das Fettsäure-Muster in den Lipiden der Nadeln von Pinus balepensis (Tab. 2) entspricht weitgehend demjenigen, das Jamieson und Reid (1972) von verschiedenen anderen Pinus-Arten beschrieben haben. Zwei Fettsäuren mit einer längeren Retentionszeit als Behensäure sind nachgewiesen, aber nicht identifiziert worden. Der Behensäure-Peak enthält sehr wahrscheinlich noch eine andere Komponente mit nahezu gleicher Retentionszeit. Wie bei den Rinden wurden Fettsäuren, die stets nur unter $1 \%$ beteiligt sind, nicht berücksichtigt. Daher fehlen in der Tab. 2 einige der bei Jamieson und Reid angegebenen Fettsäuren.

Table 2: Fatty acid composition of saponifiable lipids from leaves of Pinus halepensis (as \% of total fatty acids) $\mathrm{Sp}=\operatorname{trace}(<0.1 \%)$.

\begin{tabular}{|c|c|c|c|c|c|c|c|c|c|c|c|c|c|}
\hline \multirow[b]{2}{*}{ fatty acid } & \multicolumn{13}{|c|}{ month } \\
\hline & 6 & 7 & 8 & 9 & 10 & 11 & 12 & 1 & 2 & 3 & 4 & 5 & 6 \\
\hline $10: 0$ and $12: 0$ & 12.8 & 7.7 & 6.3 & 6.5 & 9.5 & 10.9 & 7.3 & 19.5 & 20.2 & 19.7 & 21.5 & 18.8 & 17.9 \\
\hline $14: 0$ & 0.7 & 2.3 & 4.8 & 1.6 & 5.2 & 3.4 & 3.0 & 2.8 & 2.1 & 3.5 & 2.1 & 2.2 & 1.9 \\
\hline $14: u$ & $\mathrm{Sp}$ & 1.6 & 1.8 & 2.0 & 1.0 & 1.4 & 2.3 & 2.5 & 1.1 & 0.4 & $S_{p}$ & 0.5 & $S_{p}$ \\
\hline $16: 0$ & 11.5 & 9.1 & 8.4 & 7.1 & 12.7 & 7.2 & 7.1 & 10.9 & 11.0 & 7.9 & 7.3 & 11.2 & 10.9 \\
\hline $16: 1$ & & & & 0.5 & 1.0 & & 0.4 & 0.4 & & & & & \\
\hline $16: 2$ & 1.3 & 2.7 & 2.6 & 0.3 & 0.3 & 3.4 & 0.2 & 0.1 & 4.2 & 2.3 & 1.3 & 3.1 & 2.0 \\
\hline $16: 3$ & & & & 3.5 & $5.6)$ & & 4.3 & 4.4 & & & & & \\
\hline $18: 0$ & 2.4 & 0.8 & 0.6 & 1.4 & 3.5 & 3.6 & 1.5 & 2.2 & 2.5 & 1.8 & 3.3 & 4.2 & 3.9 \\
\hline $18: 1$ & 5.0 & 4.0 & 3.3 & 4.6 & 6.6 & 6.1 & 5.9 & 6.4 & 7.1 & 4.8 & 4.9 & 5.0 & 5.6 \\
\hline $18: 2$ & 7.2 & 4.3 & 3.1 & 4.5 & 13.2 & 11.8 & 7.8 & 9.0 & 8.6 & 6.1 & 7.7 & 7.9 & 9.5 \\
\hline 18:3 (2 peaks) & 19.5 & 16.4 & 15.9 & 14.5 & 18.1 & 25.2 & 19.9 & 22.4 & 22.9 & 21.1 & 24.5 & 20.2 & 22.3 \\
\hline $18: 4$ & 4.0 & 5.4 & 4.5 & 6.3 & 2.2 & 2.6 & 7.5 & 2.2 & 2.4 & 5.3 & 2.0 & 2.3 & 3.2 \\
\hline $20: 0$ and $20: 1$ & 0.9 & 0.9 & 0.7 & 0.9 & 1.2 & 1.3 & 1.5 & 1.4 & 1.0 & 1.1 & 1.1 & 1.0 & 0.8 \\
\hline $20: 2$ & 3.1 & 3.1 & 2.8 & 3.0 & 0.9 & 0.9 & 1.1 & 0.9 & 0.7 & 0.8 & 0.9 & 0.6 & 3.0 \\
\hline $20: 3($ & 11.5 & 9.2 & 8.6 & 7.5 & 7.0 & 14.3 & 12.6 & 7.1 & 11.5 & 14.3 & 12.6 & 12.6 & 7.3 \\
\hline $20: 4$ & 3.0 & 4.9 & 4.7 & 4.7 & 4.0 & 2.9 & 3.9 & 2.4 & 1.9 & 2.0 & 1.8 & 1.7 & 2.2 \\
\hline $22: 0$ & 5.5 & 9.5 & 10.8 & 11.1 & 4.1 & 3.2 & 4.2 & 3.0 & 1.1 & 3.0 & 2.8 & 3.9 & 3.1 \\
\hline $\begin{array}{l}\text { not Identified } \\
(3 \text { peaks })\end{array}$ & 11.5 & 18.1 & 21.1 & 20.0 & 3.9 & 1.8 & 4.9 & 2.4 & 1.7 & 5.9 & 6.2 & 4.8 & 4.5 \\
\hline
\end{tabular}

Die Schwankungen der Fettsäure-Gehalte in den Nadeln sind generell etwas unregelmäßiger als in den Rinden. Jedoch sind auch hier einige Fettsäuren während der Sommermonate und zum Teil bis in den Spätherbst deutlich vermehrt (Eikosadien- und -tetraensäure, Behensäure), andere nehmen ab Oktober stark zu und haben ihre Maxima im Winter und während der Hauptwachstumszeit. Unter diesen haben die Linolensäure als mengenmäßig wichtigste Fettsäure sowie die kurzkettigen Säuren Caprin- und Laurinsäure die größte Bedeutung. Prozentuale Maxima während der kühleren Jahreszeit haben aber auch die anderen ungesättigten $\mathrm{C}_{18}$-Säuren, ungesättigte $\mathrm{C}_{16}$-Säuren sowie Eikosatriensäure. Die Mengenzunahme der Linolensäure 
erfolgt im Herbst langsamer als diejenige der Linolsäure und erst nach Einsetzen höherer Niederschläge. Berücksichtigt man bei der Berechnung der prozentualen Fettsäure-Gehalte die nur während der Sommermonate stark ins Gewicht fallenden nicht identifizierten Fettsäuren nicht, so findet man auch für Myristinsäure, Palmitinsäure und Oktadekatetraensäure deutlich erhöhte Anteile während der trockenen Sommermonate.

\section{Schinus molle}

a) Rinde

Uber die zahlreichen Fettsäuren der Lipide aus den Rinden von Schinus unterrichtet Tabelle 3. Unter « $\langle 14: 0$ » sind mindestens 4 kürzerkettige Fettsäuren zusammengefaßt (Caprin- und Laurinsäure sowie ungesättigte Fettsäuren). Die als "24:u» bezeichnete Fettsäure ist wahrscheinlich eine ungesättigte $C_{24}$-Fettsäure; nicht auszuschließen ist jedoch, daß es sich um eine hoch ungesättigte $C_{22}$-Säure handelt.

Table 3: Fatty acid composition of saponifiable lipids from bark of Schinus molle (as \% of total fatty acids).

\begin{tabular}{|c|c|c|c|c|c|c|c|c|c|c|c|c|c|}
\hline \multirow[b]{2}{*}{ fatty acid } & \multicolumn{13}{|c|}{ month } \\
\hline & 6 & 7 & 8 & 9 & 10 & 11 & 12 & 1 & 2 & 3 & 4 & 5 & 6 \\
\hline$<14: 0$ & 2.1 & 2.4 & 8.3 & 4.0 & 3.3 & 4.1 & 2.2 & 1.6 & 2.9 & 5.4 & 2.1 & 2.9 & 3.3 \\
\hline $14: 0$ & 1.2 & 1.8 & 2.2 & 3.2 & 1.6 & 2.8 & 1.0 & 1.1 & 1.6 & 1.7 & 1.3 & 3.4 & 1.1 \\
\hline $16: 0$ & 13.0 & 16.3 & 17.1 & 19.8 & 12.0 & 9.7 & 5.8 & 9.7 & 9.9 & 9.1 & 11.2 & 12.9 & 13.2 \\
\hline $16: u$ & 0.7 & 1.0 & 1.5 & 1.2 & 1.3 & 0.6 & 0.7 & 0.6 & 0.9 & 1.1 & 0.4 & 1.0 & 0.3 \\
\hline $18: 0$ and $18: 1$ & 5.8 & 4.7 & 8.0 & 9.3 & 9.1 & 5.3 & 3.0 & 2.5 & 4.5 & 3.2 & 3.5 & 4.4 & 2.3 \\
\hline $18: 2$ & 14.7 & 16.3 & 19.7 & 26.5 & 17.1 & 13.1 & 11.9 & 16.5 & 18.9 & 14.9 & 18.2 & 23.7 & 11.7 \\
\hline $18: 3$ & 11.5 & 6.7 & 6.2 & 4.1 & 10.4 & 4.3 & 4.0 & 4.9 & 6.8 & 5.3 & 9.0 & 10.1 & 11.3 \\
\hline $20: 3$ (and 20:2?) & 5.2 & 6.0 & 2.2 & 3.1 & 5.3 & 3.2 & $3.1\}$ & & & 4.4 & & & 7.9 \\
\hline $20: 4$ & 1.0 & 2.2 & 1.9 & 0.9 & 3.6 & 3.6 & 1.2 & & & 3.8 & & & 1.9 \\
\hline $22: 0$ & 3.9 & 6.0 & 2.0 & 5.0 & 4.6 & 16.7 & 20.5 & 12.2 & 10.9 & 7.4 & 7.8 & 3.6 & 3.4 \\
\hline $22: 1$ & 7.2 & 12.9 & 1.4 & 2.0 & 2.1 & 5.8 & 9.3 & 9.7 & 6.9 & 7.7 & 7.8 & 9.6 & 10.0 \\
\hline $24: 0$ & 10.7 & 8.7 & 7.2 & 12.2 & 10.0 & 14.2 & 19.3 & 19.5 & 19.1 & 17.1 & 19.6 & 14.4 & 11.5 \\
\hline $24: u$ & 16.8 & 10.4 & 15.4 & 6.6 & 11.7 & 10.0 & 14.0 & 14.1 & 10.2 & 10.1 & 10.7 & 9.1 & 15.1 \\
\hline $\begin{array}{l}\text { not identified } \\
(3 \text { peaks })\end{array}$ & 6.2 & 4.8 & 6.9 & 2.1 & 7.9 & 3.0 & 0.9 & 2.4 & 2.1 & 8.8 & 4.3 & 1.6 & 7.0 \\
\hline
\end{tabular}

Die meisten Fettsäuren weisen ausgeprägte jahresperiodische Schwankungen auf. Während der kühlen Jahreszeit und der Wachstumsphase erhöhte Anteile haben Linolensäure, Erucasäure und Lignocerinsäure. Hingegen sind Palmitinsäure, Stearinund Olsäure, Linolsäure und die als « $24: u$ » bezeichnete Säure während der trockenen Sommerperiode vermehrt. 
Table 4: Fatty acid composition of saponifiable lipids from leaves of Schinus molle (as \% of total fatty acids) $\mathrm{Sp}=$ trace $(<0.1 \%)$.

\begin{tabular}{|c|c|c|c|c|c|c|c|c|c|c|c|c|c|}
\hline \multirow[b]{2}{*}{ fatty acid } & \multicolumn{13}{|c|}{ month } \\
\hline & 6 & 7 & 8 & 9 & 10 & 11 & 12 & 1 & 2 & 3 & 4 & 5 & 6 \\
\hline$<14: 0$ & 6.2 & 10.3 & 6.6 & 7.9 & 6.8 & 7.1 & 5.1 & 6.4 & 6.2 & 6.3 & 4.9 & 2.2 & 4.3 \\
\hline $14: 0$ & 3.1 & 2.2 & 1.2 & 2.5 & 1.6 & 2.8 & 1.8 & 3.1 & 2.2 & 3.0 & 2.0 & 1.8 & 1.8 \\
\hline $14: u$ & $S_{p}$ & 1.8 & Sp & 0.6 & 0.3 & 0.4 & 0.2 & Sp & $\mathrm{Sp}$ & $\mathrm{Sp}_{\mathrm{p}}$ & $S_{p}$ & $\mathrm{Sp}_{\mathrm{p}}$ & $S_{p}$ \\
\hline $16: 0$ & 12.1 & 9.4 & 10.0 & 12.9 & 6.8 & 9.8 & 6.6 & 8.7 & 7.2 & 12.1 & 10.6 & 12.2 & 7.6 \\
\hline $16: u$ & 1.0 & 2.2 & 2.4 & 2.6 & 2.2 & 0.9 & 0.2 & 0.7 & 0.7 & 1.3 & 0.7 & 1.1 & 0.7 \\
\hline 18:0 & 0.4 & 1.1 & 0.4 & 0.9 & 0.8 & 0.8 & 0.5 & 0.7 & 0.5 & 0.6 & 0.6 & 0.5 & 0.4 \\
\hline 18:1 & 4.0 & 4.9 & 4.1 & 5.5 & 4.1 & 3.4 & 2.0 & 2.0 & 1.7 & 2.5 & 1.3 & 2.9 & 2.3 \\
\hline $18: 2$ & 6.3 & 5.6 & 6.4 & 6.1 & 5.1 & 7.5 & 5.6 & 8.3 & 7.2 & 9.3 & 6.7 & 10.6 & 6.3 \\
\hline $18: 3$ & 13.5 & 11.9 & 8.8 & 13.0 & 11.3 & 13.2 & 17.9 & 15.9 & 10.8 & 14.9 & 9.7 & 17.8 & 12.3 \\
\hline $20: 2$ and $20: 3$ & 7.9 & 4.9 & 3.2 & 4.0 & 3.6 & $\mathrm{Sp}$ & $\mathrm{Sp}$ & 4.1 & 1.8 & 1.4 & 2.0 & 1.4 & 6.0 \\
\hline $20: 4$ & 1.6 & - & 2.6 & 2.5 & 2.9 & - & 1.1 & 1.6 & 2.2 & - & 2.9 & - & 1.4 \\
\hline $22: 0$ & 3.9 & 4.7 & 3.5 & 2.3 & 7.9 & 6.3 & 6.2 & 6.5 & 8.3 & 9.7 & 9.1 & 7.3 & 6.0 \\
\hline $22: 1$ & 4.6 & 4.7 & 5.9 & 2.8 & 9.9 & 3.8 & 3.4 & 5.1 & 7.6 & 5.4 & 6.3 & 5.3 & 4.8 \\
\hline $24: 0$ & 14.6 & 13.0 & 17.1 & 13.5 & 19.5 & 19.1 & 20.5 & 16.6 & 20.0 & 19.8 & 16.6 & 17.3 & 17.7 \\
\hline $24: u$ & 15.5 & 15.7 & 27.7 & 15.3 & 14.7 & 22.2 & 24.9 & 14.5 & 16.1 & 12.0 & 18.2 & 13.9 & 24.2 \\
\hline $\begin{array}{l}\text { not identifted } \\
\text { ( } 2-3 \text { peaks) }\end{array}$ & 53 & 7.6 & 4.1 & 7.6 & 2.5 & 2.3 & 4.0 & 5.8 & 7.5 & 1.7 & 8.4 & 5.7 & 4.2 \\
\hline
\end{tabular}

b) Blätter

Das Fettsäure-Muster der Blatt-Lipide von Schinus geht aus Tabelle 4 hervor. Einer der nicht identifizierten Fettsäure-Peaks geht sehr wahrscheinlich auf Hexakosansäure (26:0) zurück.

Unter den Fettsäuren werden im Sommer vor allem die ungesättigten $\mathrm{C}_{16}$-Säuren sowie Olsäure vermehrt; hingegen sind die Anteile an Linol- und Linolensäure sowie an Behen- und Lignocerinsäure während der Winter- und Frühjahrsmonate erhöht. Palmitinsäure erreicht die größten Anteile während der Wachstumszeit und zu Beginn des Sommers.

\section{Diskussion}

Die Jahresperiodik der meisten Fettsäuren der Rindenlipide ist in beiden Arten ähnlich. Stearin-, Ol- und Linolsäure werden allerdings bei Schinus im Sommer, bei Pinus aber im Winter vermehrt. In den Blattlipiden verhalten sich vor allem Linolund Linolensäure bei beiden Arten gleichartig; der Anteil nimmt während der kühlen und zugleich feuchten Jahreszeit zu. Eine Vermehrung ungesättigter Fettsäuren während des Winters wird in der Literatur insbesondere von grünen Geweben vielfach beschrieben (z. B. Kuiper, 1970; de le Roche et al., 1975; Willemot et al., 1977; Ketchie und Kuiper, 1979). Willemot und Pelletier (1980) haben bei Weizen gezeigt, daß bei niedriger Temperatur und hinreichend Licht die Linolensäure vermehrt 
wird; Beziehungen zur Entwicklung der Frosthärte bestehen dabei nicht (vgl. auch de la Roche, 1979). Eine besonders starke Zunahme der Linolensäure nach Eintritt der feuchten Jahreszeit in den Pinus-Nadeln könnte mit einer Reaktivierung der Chloroplasten zusammenhängen. Linolensäure ist ja vorwiegend in den Glykolipiden der Chloroplasten lokalisiert und Peoples et al. (1978) haben gezeigt, daß zwischen dem Anteil ungesättigter Fettsäuren der Chloroplastenmembran und der Photosyntheseleistung Beziehungen bestehen. In den Pinus-Nadeln werden in der kühlen Jahreszeit auch die kurzkettigen Fettsäuren stark vermehrt. Sofern diese Fettsäuren Bausteine von Membranlipiden sind, wäre auch dadurch eine Regulation der Membranfluidität möglich.

In den Rinden sind die Anteilsveränderungen der ungesättigten Fettsäuren eher unregelmäßig. Dies entspricht Befunden bei Arten des kühler gemäßigten Klimas (Yoshida und Sakai, 1974; Siminovitch et al., 1975).

Die sommerliche Trockenheit hat bei beiden untersuchten Arten auf die Fettsäurezusammensetzung der Lipide keinen deutlichen Einfluß. Dies stimmt mit den Befunden von Kull und Breckle (1975) an Steppenpflanzen und den Untersuchungen von Diamantoglou und Meletiou-Christou $(1977,1979)$ an Ceratonia und Pistacia überein.

Pinus und Schinus führen beide Harz. Wie schon erwähnt, könnten die sehr langkettigen Fettsäuren teilweise aus Harzen oder Wachsen stammen. Allerdings zeigten Jamieson und Reid (1972), daß bei Pinus auch die Phospho- und Glykolipide Fettsäuren zumindest bis zu 22C-Atomen enthalten. Die Berechnung der prozentualen Anteile der Fettsäuren bei Nichtberücksichtigung der Fettsäuren mit mehr als 20C-Atomen führt nicht zu wesentlichen Veränderungen in der Jahresperiodik der übrigen Fettsäuren; allerdings treten einige Effekte etwas klarer heraus.

Im Harz der Früchte von Schinus sind Fettsäuren bis zu 28C-Atomen nachgewiesen worden (Pozzo-Balbi, 1978); die hierbei mengenmäßig wichtigste Lignocerinsäure hat auch in den Blatt-Lipiden einen erheblichen Anteil. Das Vorkommen zahlreicher Fettsäuren mit mehr als 20C-Atomen bei Schinus ist ein weiteres Beispiel für die merkwürdige biochemische Konvergenz von Vertretern der Anacardiaceae mit Gymnospermen (Hegnauer, 1964). Allerdings weicht das physiologische Verhalten gerade der sehr langkettigen Fettsäuren bei Pinus und Schinus zum Teil voneinander $a b$. Die bezüglich der Stoffe offensichtliche Konvergenz gilt also für deren Stoff wechsel nur eingeschränkt.

Frau Eva-Maria Moser danken wir für sorgfältige Mitarbeit; der Deutschen Forschungsgemeinschaft für eine Sachbeihilfe (an U.K.).

\section{Literatur}

Catalano, M. e V. Sciancalepore: Riv. Ital. Sostanze Grasse 52, 276-280 (1975).

De La Roche, I. A.: Plant Physiol. 63, 5-8 (1979).

De La Roche, I. A., M. K. Pomeroy, and C. J. Andrews: Cryobiology 12, 506-512 (1975).

Diamantoglou, S.: Ber. dtsch. Bot. Ges. 93, 449-457 (1980).

Z. Pflanzenphysiol. Bd. 103. S. 157-164. 1981. 
Diamantoglou, S. und M. S. Meletiou-Christou: Z. Pflanzenphysiol. 85, 95-101 (1977). - - Z. Pflanzenphysiol. 93, 219--228 (1979).

Donaire, J. P. and J. Lopez-Gorgé: J. exp. Bot. 29, 49-54 (1978).

Hegnauer, R.: Chemotaxonomie der Pflanzen, Band 3, Basel und Stuttgart (1964).

Höllwarth, M. und U. Kull: Z. Pflanzenphysiol. 91, 325-331 (1979).

Jamieson, G. R. and E. H. Reid: Phytochemistry 11, 269-275 (1972).

Ketchie, D. O. and P. J. C. Kuiper: Physiol. plant. 46, 93-96 (1979).

Kuiper, P. J. C.: Plant Physiol. 45, 684-686 (1970).

Kull, U. und K. Jeremias: Z. Pflanzenphysiol. 68, 55-62 (1972).

Kull, U. und S.-W. Breckle: Z. Pflanzenphysiol. 75, 332-338 (1975).

Peoples, T. R., D. W. Koch, and S. C. Smith: Plant Physiol. 61, 472-473 (1978).

Porter, L. J.: New Zeal. J. Sci. 12, 687-693 (1969).

Pozzo-Balbi, T., L. Nobile, G. Scapini, and M. Cini: Phytochemistry 17, 2107-2110 (1978).

Siminovitch, D., J. Singh, and I. De La Roche: Cryobiology 12, 144-153 (1975).

Wiedmaier, J. und U. Kull: Biochem. Physiol. Pflanzen 172, 421-437 (1978).

Willemot, C., H. J. Hope, R. J. Williams, and R. Michano: Cryobiology 14, 87-93 (1977).

Willemot, C. et L. Pelletier: Canad. J. Plant Sci. 60, 349-355 (1980).

Yoshida, S. and A. Sakai: Plant Physiol. 53, 509-511 (1974). 